\title{
Determination of the efficiency of the AM2 dehairing technology process with Llama fiber of different types of fleeces and Alpaca Huacaya fiber
}

\begin{abstract}
The dehairing process is a fiber cleaning process and consists of separating objectionable coarse fibers and plant and mineral contaminants from the fine fibers as efficiently as possible. This efficiency is determined by the fiber type removal/retention rate (RR) and the fiber length shortening rate (FL). Up the present, in fiber from Llamas and Alpacas, the dehairing yield $(\mathrm{R} \%$ ) has been used as a commercial evaluation of the efficiency of the process. Efficiency is of greater commercial importance because it reflects the amount of desirable fiber that is actually obtained after the cleaning process has been performed. It also allows comparing technologies, number of passages and process costs. The objective of this work is to determine the efficiency of Llama and Huacaya Alpaca fiber dehairing carried out by AM2 technology based on the retention/removal (RR) and shortening (FL), taking into consideration different types of fibers (GF), types of fleece (TV), using methodology used in other fibersusing methodology and variables from studies with other fibers. The dehairing efficiency of the AM2 technology is similar in Llama and Alpaca fiber and slightly better than a similar technology used in cashmere fiber in Australia. In general efficiency fluctuates between $74-94 \%$, in terms of desirable fine fiber recovery, for the textile process of these fibers. The variable defined as the fiber type removal/retention rate, is usable to correctly evaluate the fiber dehairing process of Llamas and Alpacas.
\end{abstract}

\author{
Volume 8 Issue I - 2022
}

\author{
Eduardo Narciso Frank, 1,2 Michel VH Hick, 1,2 \\ María Flavia Castillo, ${ }^{2}$ Diego Seghetti \\ Frondizi, ${ }^{3}$ Lilian Irene Brodtmann ${ }^{3}$ \\ 'IRNASUS, CONICET-UCC, Catholic University of Cordoba, \\ Argentina \\ ${ }^{2}$ Sede Chamical-UNLAR, Argentina \\ ${ }^{3}$ Private entrepreneurs, Argentina
}

\begin{abstract}
Correspondence: Eduardo N Frank, Designation Professor, PhD, IRNASUS, CONICET-UCC, Catholic University of Cordoba, UNLAR, Armada Argentina 3555. Córdoba, Argentina, Tel 54 935I 4938030,Email frank.agro@ucc.edu.ar
\end{abstract}

Received: January 25, 2022 | Published: February 17, 2022

Keywords: fiber, textile, dehairing, cashmere

\section{Introduction}

The textile processing of animal fibers to which they belong to the group of special fibers (goat and camelid fibers), requires a cleaning process, also called ennoblement, prior to the start of carding and subsequent processes, until reaching the yarn.

The dehairing process is precisely a cleaning process and consists of separating objectionable coarse fibers and plant and mineral contaminants from the fine fibers as efficiently as possible. ${ }^{1}$ This efficiency is determined by the fiber type removal/retention rate (RR) and the fiber length shortening rate (FL). ${ }^{2}$ Until now, in fiber from Llamas and Alpacas, the dehairing yield (R\%) has been used as a commercial evaluation of the efficiency of the process, as defined by Singh (2003). ${ }^{1}$ Only studies on cashmere fiber have been carried out with this parameterized variable. ${ }^{2}$

In a study with raw cashmere it was investigated the effects of attributes on the efficiency of dehairing. The results show that an more efficient processing and/or the production of longer cashmere were associated with the following attributes of raw cashmere: white color, longer raw cashmere, greater fiber curvature, lower vegetable matter, normal length guard hair, absence of visible felted. ${ }^{3}$ A similar experience was not carried out with fibers from domestic South American Camelids (SAC) in South America.

On the other hand, this textile pre-process is essential for raw cashmere and has been internationally used for alpaca, llama, camel and yak. ${ }^{4}$ Results of dehairing Alpaca fiber, pre-processed at top level (carding, gilling and combing) was successful. In this case, Objectionable reducing coarse fibers can reach the minimum required $(<3.2 \%)$ not to cause prickle possible effect at six runs of the process. Despite the length reduction by fiber breakage, it is still possible to process the final product by the worsted system. ${ }^{5}$
In more recent study, with Argentine Llama fiber, a differential behavior of the dehairing process was determined, according to the types of fibers (FT) and/or according to the types of fleece from which the samples came. ${ }^{6}$

However, the economic evaluation of the process is still under way and the feasibility of improving the price of Alpaca fiber by dehairing is subject to this assessment, and the efficiencies of the process is decisive.

Although the dehairing yield is used commercially as an indication of the performance of the process, it depends on factors that are inherent to the fiber itself and not so much to the technology. ${ }^{7}$

The objective of this work is to determine the efficiency of Llama and Huacaya Alpaca fiber dehairing carried out by AM2 technology based on the retention/removal (RR) and shortening (FL) taking into consideration the different types of fibers (GF), types of fleece (TV), using methodology and variables from studies with other fibers.

\section{Materials and methods}

Sixteen $(n=16)$ fleeces of different types (TV) were used: double coat (DC: 5), single coat (SC: 8), luster (L: 3) ${ }^{8}$ Super Fine (SF): 3 , Fine (f): 8 , Medium (M): 3; and coarse (G): 2 and shearing interval (Int.Es): annual (9) and biannual (7).

The belly and matted (cotted) parts of the fleece were separated. Humidification and addition of antistatic agent were carried out and 10 passes ( $\left.\mathrm{N}^{\circ} \mathrm{Pas}\right)$ were made through a dehairing module of the AM2 equipment, ${ }^{7}$ establishing the $7^{\text {th }}$ pass with minimal coarse fiber removal (FG1) (Figure 1). ${ }^{11}$

The outputs of each passage (product and residuals) adjusted to constant humidity were weighed and in the LAFTA laboratory, 
were dissected and separated in the 3 groups of fibers: coarse (FG1), intermediate (FG2) and fine (FG3). The dehairing yield (R\%) of each pass, the retention/removal efficiency (RET\%) and the mean fiber diameter (DMF) and mean fiber length (LF) were determined. In the case of the dehaired residuals, the accumulated fiber weight per passage and the accumulated fiber removal were established.

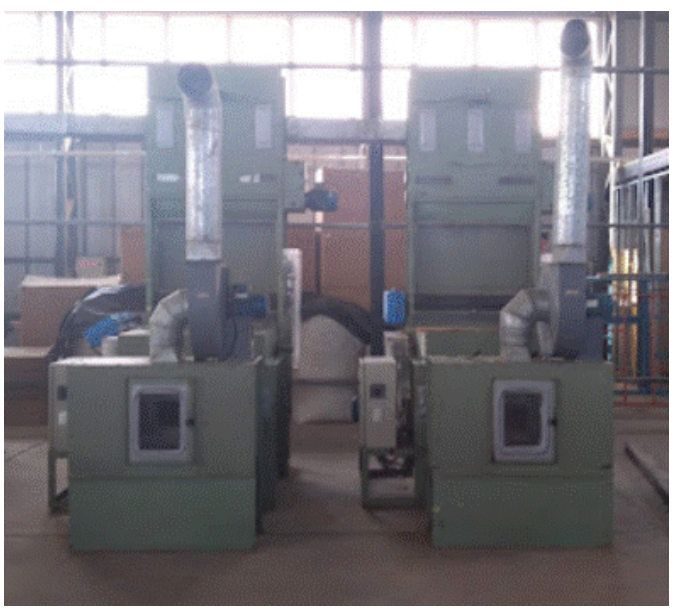

Figure I A panoramic view of two AM2 modules operating in a textile plant.

\section{Statistical analysis}

Data in Tables are expressed as Confidence Intervals (CI). These confidence intervals have a designed probability of containing the true mean of $95 \%$ or greater. The parameterization of the efficiency variables allows expressing as CI to decimate a null hypothesis when comparing with a similar result obtained in another technology or another circumstance of the same. For hypothesis tests, if the $\mathrm{CI}$ included the mean, it would imply that for $\mathrm{p}<0.05$, at $5 \%$ probability, the null hypothesis would not be rejected. ${ }^{9}$ They were estimated using the IC Generation Calculator of the statistical program InfoStat. ${ }^{9}$

The mean comparison of variables of the dehaired fiber were compared by ANOVA of the Generalized Mixed Linear Models and comparison method cluster-based means of DGC was used. ${ }^{9}$ In the case of Scatter plot graphics, the means were adjusted by Loewess. ${ }^{9}$

\section{Results and discussion}

At ANOVA, the frequencies of fibers by type of shearing and the interaction were not significant for RET\%, and also for $\mathrm{FGxN}^{\circ}$ Pass $(p>0.05)$. A similar behavior was observed in FL, despite the interaction with shearing type, which was $1.6 \mathrm{~cm}$ longer in favor of biannual shearing. This is so, despite the fact that the effect of nonannual shearing times on dehairing efficiency is discussed. ${ }^{6}$

Table 1 shows the frequency changes of the fiber types at the seventh pass (7th M\&M), in all cases the lustre Type of Fleece (TV L) was different from the others according to Frank et al., ${ }^{10}$ and the frequencies were similar to the 6th pass on top of Alpaca. ${ }^{5}$ The luster fleece type (Suri for Alpaca) has a higher coarse fiber content $(>30 \mu \mathrm{m})$ by laboratory analysis, but not visible as objectionable fibers to the naked eye. ${ }^{10}$

The R\% separated by fineness at the 7th pass gave: super fine (SF): $65 \%$; fine $(\mathrm{F}): 60 \%$; medium $(\mathrm{M}): 60 \%$ and coarse $(\mathrm{G}): 45 \%$, while for type of fleece (TV): DC: $41 \%$; SC: $63 \%$ and L: $50 \%$. The yield in fine goat fiber (R\%) at the 7th pass was between: 49.3-60.0 (95\%) with an average of $54.7 \%$, while in cashmere content it was between: 38.0 $31.4 \%{ }^{2}$ Visually, the Llama and Alpaca fibers have a less felted aspect than the cashmere fiber, this could explain the difference.
Table I Top: Initial frequencies of fiber types (FGI, FG2, FG3); Bottom fiber frequencies after passage $7^{\text {th }}$ in relation to types of fleeces (TV) from Llama fibre

\begin{tabular}{|c|c|c|c|c|c|c|c|c|c|}
\hline \multicolumn{10}{|c|}{0 Passage } \\
\hline \multicolumn{3}{|c|}{ FG I } & & \multicolumn{3}{|c|}{ FG2 } & \multicolumn{2}{|c|}{ FG3 } & \\
\hline TV & $5 \%$ & $95 \%$ & & $5 \%$ & $95 \%$ & & $5 \%$ & $95 \%$ & \\
\hline DC & 20,2 & 22,8 & $b$ & 14,0 & 15,2 & $b$ & 62,0 & 65,7 & $\mathrm{~b}$ \\
\hline SC & 11,1 & 30,8 & c & 6,0 & 16,8 & $a$ & 60,4 & 80,3 & $\mathrm{~b}$ \\
\hline L & 40,4 & 50,6 & $\mathrm{a}$ & 9,9 & 10,2 & $\mathrm{a}$ & 39,5 & 49,3 & $\mathrm{a}$ \\
\hline \multicolumn{10}{|c|}{7 th Passage } \\
\hline DC & 2,6 & 2,9 & $b$ & 2,2 & 2,3 & $\mathrm{a}$ & 67,6 & 71,6 & $\mathrm{a}$ \\
\hline SC & $\mathrm{I}, 4$ & 3,9 & $b$ & 0.8 & 2,1 & $\mathrm{a}$ & 72,4 & 96,3 & $\mathrm{a}$ \\
\hline L & 9,2 & 11,5 & $\mathrm{a}$ & 2,9 & 1,5 & $\mathrm{a}$ & 43,1 & 53,7 & b \\
\hline
\end{tabular}

*equal letters in columns mean non-significant differences between means $(p>0.05)$

Efficiency in RET\% (Figure 2) is slightly higher than R\% for each GF, this does not agree with cashmere where is $10.5 \%$ higher than the $\mathrm{R} \% .^{2}$ This would show a better indication of objectionable fibers removed, while $\mathrm{R} \%$ also shows vegetables and soil removed.

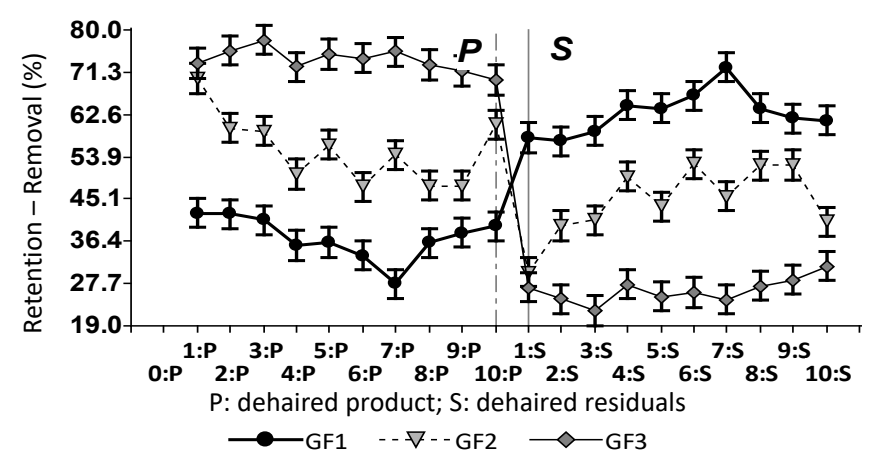

Figure 2 Efficiency expressed as Retention (GF3), Removal (GFI), Retention/ Removal (GF2), expressed as a percentage of the weight of the original nondehaired Llama fiber.

The fibre length (FL) Confidence Interval (CI) was: lower 5\%: $7.9 \mathrm{~cm}$, upper $95 \%: 8.5 \mathrm{~cm}$ and the $\mathrm{LF} / \mathrm{LM}$ ratio ranges from 0.62 to 0.79 . While for cashmere a CI of: lower5\% was obtained: 2.4 to upper. $95 \%: 7.4 \mathrm{~cm}^{2}$

What is interesting is the LF/length ratio, initial staple length between the lower 5\%: 0.62 to upper 95\%: 0.79, were in cashmere that relationship was lower: $0.48-0.64 .^{2}$

Recalculating the results obtained in Cashmere in a previous work yields a similar result. ${ }^{11}$ This would be demonstrating an improved behavior of the fiber of Llama and Alpaca to the shortening of the fiber, not necessarily due to the technology used.

Table 2 shows the frequency changes of the fiber types between the raw fiber and the 5 th pass of dehairing. Although it starts from a lower level than the Llama fiber in general, it behaves very similar to the SC fleece type. It is striking here because Alpaca fiber a higher level of GFR reduction is achieved than in the 6 th pass of a previous work. ${ }^{5}$

Figure 3 shows the performance of the Alpaca Huacaya fiber when dehairing. The product's dehairing performance is similar to that of the Llama, but lower levels are achieved in the dehairing 
residue, marking a slightly higher efficiency than in SC Llama type (96.95-97.85 vs $72.4-96.3 \%$ ). This is possibly due to the differences in the presentation of the raw fleece in the Llama with respect to the Alpaca. ${ }^{2}$ The Llama fleeces, in addition to the three types of fleece, also belonged to different finenesses, while the Alpaca was only of the Huacaya type and with a superfine fineness.

Table 2 Initial frequencies of fiber types (FGI,FG2, FG3) and fiber frequencies after passage $5^{\text {th }}$ in relation to types of fleeces Huacaya from Alpaca fibre

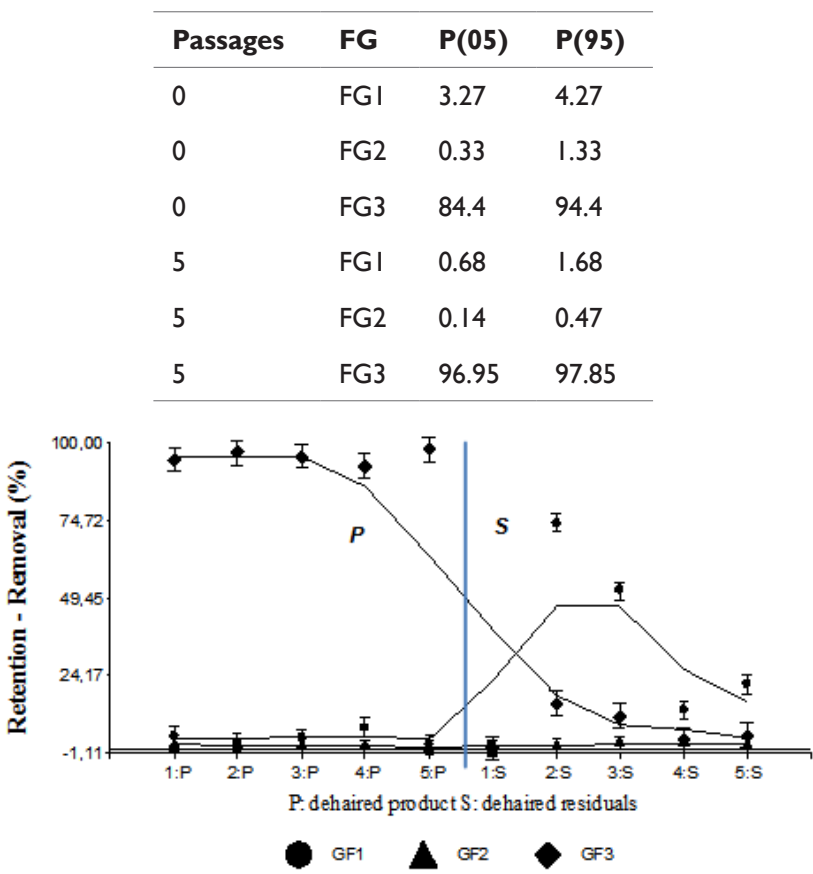

Figure 3 Efficiency expressed as Retention (GF3), Removal (GFI), Retention/ Removal (GF2), expressed as a percentage of the weight of the original nondehaired Alpaca fiber

\section{Conclusion}

The dehairing efficiency of the AM2 technology is similar in Llama and Alpaca fiber and slightly better than a similar technology used in cashmere fiber in Australia. In general efficiency fluctuates between $74-94 \%$, in terms of desirable fine fiber recovery, for the textile process of these fibers.

The variable defined as the fiber type removal/retention rate, is usable to correctly evaluate the fiber dehairing process of Llamas and Alpacas.

\section{Acknowledgments}

None.

\section{Funding}

None.

\section{Conflicts of interest}

Authors declare that there is no conflict of interest.

\section{References}

1. Singh A. A study o f dehairing of Australian cashmere fibres. Deakin University, Master Thesis. 2003.

2. Mcgregor BA. Scouring and Dehairing Austrlian Cashmere. Agri Futurres Goat Fibre. 2018. 34 p.

3. Mcgregor BA, Butler KL. The effects of cashmere attributes on the efficiency of dehairing and dehaired cashmere length. Text Res J. 2008;78:486-496.

4. Mcgregor BA. Properties, processing and performance of rare and natura fibres. A review and interpretation of existing research results. RIRDC Res Rep. 2012.

5. Frank EN, Seghetti Frondizi DG, Hick MHV, et al. Results of dehairing process on alpaca fibres with a new dehairing technology. Int J of Polymer and Text Engen. 2019;6(1):1-3.

6. Brodtmann LI. Effect of age on the structure of the llama fleece and its relationship with the textile quality of the fiber. Doctoral Thesis Agricultural Science, Catolic Univ of Cordoba; 2020.

7. Seghetti Frondizi DG. The dehairing of fibers for the textile industry from camelids and other double-layer species. Trabajo final UB; 2009.

8. Frank EN, Hick MHV, Castillo MF, et al. Effect on textile behavior of fleece types and dehairing process on the linear density and regularity of yarn from argentine llama fibre. Journal of Textile Science \& Fashion Technology. 2019;2(2):1-3.

9. InfoStat. InfoStat versión 1.1. Grupo InfoStat, FCA, Universidad Nacional de Córdoba, Argentina. 2002.

10. Frank EN, Hick MVH, Adot O. Descriptive differential attributes of type of fleeces in Llama fibre and its textile consequence: 1 - Descriptive aspects. $J$ The Text Inst. 2007;98(3):251-259.

11. Frank EN, Hick MVH, Castillo MF, et al. Determination of the optimal number of runs of dehairing in fibers of patagonian cashmere goats. $J$ Textile Eng Fashion Technol. 2018;4(3):188-190. 